\title{
Leukocyte count and the risk of adverse outcomes in patients with HFpEF
}

\author{
Zhaowei Zhu and Shenghua Zhou* (D
}

\begin{abstract}
Background: Inflammation is a key feature of heart failure including HFpEF. The leukocyte count is a marker of inflammation that is widely used in clinical practice. However, there is little available evidence for the relationship between leukocyte count and the outcomes of HFpEF.

Methods: We analyzed data from the TOPCAT (Treatment of Preserved Cardiac Function Heart Failure with an Aldosterone Antagonist) trial. The primary outcome was all-cause mortality, the secondary outcome was composite cardiovascular events and hospitalization for heart failure. Multivariable Cox proportional hazard models were used to compare the risk profiles of patients with leukocyte quartiles, subgroup study divided by sex was also analyzed.

Results: The present study included 2898 patients with HFpEF.429 deaths, 671 composite cardiovascular events and 386 hospitalization for heart failure occurred during a mean 3.4 years follow-up. The association between leukocyte count and adverse outcomes followed a U-shaped curve. After multivariable adjustment, the patients with the lowest leukocyte count (Q1) and the highest leukocyte count (Q4) faced higher risk of all-cause death(Q1 vs. Q2, adjusted HR: $1.439 ; 95 \% \mathrm{Cl}: 1.060-1.953, \mathrm{p}=0.020 ; \mathrm{Q} 4$ vs. Q2, adjusted HR, 1.901; 95\%Cl: $1.424-2.539, \mathrm{p}<0.001)$. The subgroup analysis showed a consistent result in female but not male patients.

Conclusions: The association between leukocyte count and risk of adverse outcomes followed a U-shaped curve. Both higher and lower leukocyte count are associated with worse outcomes in patients with HFpEF, which may be attributed to the two sides of inflammation in cardiac remodeling.
\end{abstract}

Keywords: HFpEF, Leukocyte, Adverse outcomes

\section{Background}

Heart failure with preserved ejection fraction (HFpEF) has emerged as anpivotal problem with increasing prevalence and poor prognosis in recent years [1]. However, it is still not fully understood of the pathophysiology of HFpEF, which retards the improvement of its accurate diagnosis and efficient treatment.In fact, proven effective medical treatment has not yet appeared for this disease $[2,3]$.

Leukocyte, as an inflammation driver, plays an important role in cardiovascular disease. In further, it even

*Correspondence: zhoushenghua@csu.edu.cn

Department of Cardiovascular Medicine, The Second Xiangya Hospital,

Central South University, Changsha 410011, Hunan, China serves as an important predictor for various cardiovascular events [4-6]. Heart failure, which is an end stage of all kinds of cardiovascular disease, has been known to be involved in inflammation process and the concept of inflammation as a major component of HF is becoming more and more consolidated [7]. Recent studie sconfirmed that inflammatory processes could be part of the etiology of HF $[8,9]$. Besides, it was shown that increased long-term incidence of HF hospitalizations were associated with high leukocyte counts [10].Moreover, subclinical inflammation predicts adverse prognosis in patients with established HF [11-13].Canakinumab (IL-1 $\beta$ inhibitor), as an inflammation inhibitor, has beenfound to be capable of reducing not only the incidence 
of hospitalization for heart failure but also heart failurerelated mortality [13].

Although limit evidences indicateinflammation biomarkers are associated with adverse outcomes in patients with HFpEF [14, 15], the relationship between leukocyte count and HFpEF is still not fully clear. Therefore, this study aimed to examine the prognostic significance of leukocyte count on clinicaloutcomes in patients with HFpEF in the Treatment ofPreserved Cardiac Function Heart FailureWith an Aldosterone Antagonist Trial(TOPCAT).

\section{Methods}

\section{Study design and patients}

TOPCAT was a randomized, placebo-control, double blind, multi-centerclinical study.The study aimed to investigate the treatment efficacy of spironolactone in patientswith HFpEF. The study information including background, design, inclusion and exclusion criteria, and baseline characteristicshave been published previously [16, 17]. Briefly, this trial, beginning in August 2006 and ending in January 2012, enrolled 3445 patientswith symptomatic HFpEF from 270 sites distributed in 6 countries. The primary goal of the trial was toclarifywhether spironolactone could reduce the compositeoutcome of aborted cardiac arrest, cardiovascular mortality, orheart failure hospitalization in patients with HFpEF (e.g. documented ejectionfraction $\geq 45 \%$ ).

According to the current guideline [18], this analysis in this investigation were limited to patients with ejectionfraction $\geq 50 \%(n=2930)$. Patients with missed leucocyte count and outlier leucocyte count (over 20,000 cells/ $\mu \mathrm{L}$ ) $(\mathrm{n}=32)$ were excluded. At last, total 2898 patients were enrolled in this study (Fig. 1).The association between leukocyte count on admission and the risk ofall-cause death, the composite cardiovascular events and hospitalization for heart failure were analyzed.

\section{Baseline characteristics}

Basic informationandmedical histories were obtained in patients by a detailed baseline visit in TOPCAT study [17]. For example, age, sex, race, and current smokers were obtained by self-reported history.Medical history included: hypertension, diabetes, stroke, dyslipidemia, peripheral arterial disease, angina pectoris, myocardial infarction, percutaneous coronary revascularization, coronary artery bypass graft surgery, implanted cardioverter defibrillator, implanted pacemaker, thyroid disease, chronic obstructive pulmonary disease, New York HeartAssociation Class, and prior heart failure hospitalization. Systolic bloodpressure, diastolic blood pressure and Body Mass Index (BMI) were obtained by trained

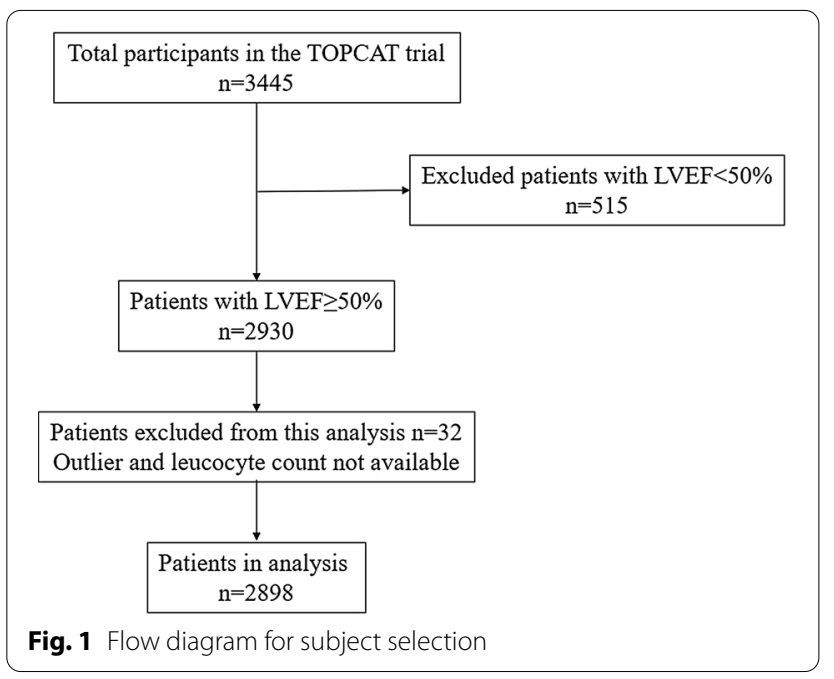

staff.Laboratorydata included serum creatinine, blood urea nitrogen (BUN), hematocrit, Brain Natriuretic Peptide (BNP), hemoglobin and platelet. Medication data included: aspirin, angiotensin-converting enzyme inhibitors/angiotensin II receptorblockers, beta blockers, calcium channel blockers, and statins.The National Heart, Lung, and Blood Institute approved our use of TOPCAT data.Ethics approval and consent toparticipate were not applicable.

\section{Statistics}

Baseline characteristics were compared by quartiles of leukocyte counts. Data are presented asmean $\pm \mathrm{SD}$, nonnormal variables were reported as median (interquartile range [IQR] - the distance between the 25th and 75th percentiles. Normally distributed continuousvariables were analyzed with one-way ANOVA. Categorical variables were compared withPearson $\chi^{2}$ test. Baseline plasma BNP levels were expressed as log-transformed data.Glomerular filtrationrates were estimated by incorporating creatinine into the ChronicKidney Disease Epidemiology Collaboration (CKD-EPI) formula [19]. UnadjustedKaplan-Meier estimates of the time-to-event outcomes were generatedaccording to baseline leukocyte countquartiles and compared via the log-rank test. Univariate and multivariable Cox regression analysis were used to test the risk of adverse outcomes associated withleukocyte count. Only variables with $\mathrm{p}<0.1$ on univariate analysis were incorporated into the multivariate Cox regression analysis. Subgroup analyses of multivariate models were done by sex. Two-sided P-values $<0.05$ were consideredstatistically significant. All analyses were performed usingEmpower(R) (www.empowerstats.com, $\mathrm{X} \& \mathrm{Y}$ solutions, IncBoston, MA) andSPSS version 25.0 (IBM, Armonk, New York). 


\section{Results}

\section{Study participants and baseline characteristics}

A total of 2898 patients (mean age $=69 \pm 9.6$ years; $46 \%$ men; $89 \%$ white) were included in this analysis. Table 1 presented participants' baseline characteristics based onleukocyte quartiles (Q):Q1: $\leqq 5.5 \times 10^{9} / \mathrm{l}$; Q2: $>5.5 \times 10^{9} / 1$ to $\leqq 6.7 \times 10^{9} / \mathrm{l} ; \quad \mathrm{Q} 3:>6.7 \times 10^{9} / \mathrm{l}$ to $\leqq 8.0 \times 10^{9} / \mathrm{l}$; and $\mathrm{Q} 4:>8.0 \times 10^{9} / \mathrm{l}$. Leukocyte quartiles were not associated with any significanttrends in age, race, prior heart failure hospitalization, hypertension, stroke, history of pacemaker or implantable cardioverter defibrillators (ICD) implanted,angina pectoris, systolic blood pressure, left ventricular ejection fraction (LVEF), heart rate, the use ofb-blockers, calcium channel blockers, angiotensin-converting enzyme inhibitor/Angiotensin Receptor Blocker (ACEI/ARB)and spironolactone. However, male sex, smoker, dyslipidemia, previous myocardial infarction, percutaneous coronary intervention (PCI), Coronary artery bypass graft (CABG), diabetes mellitus, atrial fibrillation, chronic obstructive pulmonary disease (COPD), asthma, thyroid disease, peripheral arterial disease, use of statins and loop diuretics were more prevalent in participants the higherleukocyte quartiles.At the same time, higher leukocytecount was associated with higher heart rate, body mass index, BUN, hemoglobin and platelet.The higher leukocyte count was also associated with lower diastolic blood pressure, eGFR and prevalence of New York Heart Association class III-IV.

\section{Leukocyte count on admission and long-term clinicaloutcomes}

Over a median follow-up of 3.4 years (25th-75thpercentiles $=2.0-4.9$ years), 429 deaths, 671 composite cardiovascular events and 386 hospitalization for heart failure occurred. Kaplan-Meier estimates of the cumulative incidence ofall-cause death, the compositecardiovascular eventsand hospitalization for heart failure are depicted in Fig. 2. It seems both participants in the highest and lowest leukocytecount quartiles faced a greater riskfor all-cause death (log-rank, $\mathrm{P}<0.0001$ forall; $\mathrm{Q} 1$ vs. $\mathrm{Q} 2$ : $\mathrm{P}<0.0001$; $\mathrm{Q} 3$ vs. $\mathrm{Q} 2: \mathrm{P}<0.0001 ; \mathrm{Q} 4$ vs. $\mathrm{Q} 2: \mathrm{P}<0.0001$ ), compositecardiovascular events(log-rank, $\mathrm{P}<0.0001$ forall; $\mathrm{Q} 1$ vs. Q2: $\mathrm{P}<0.0001 ; \mathrm{Q} 3$ vs. Q2: $\mathrm{P}<0.0001$; 4 4s. Q2: $\mathrm{P}<0.0001$ )and hospitalization for heart failure (logrank, $\mathrm{P}<0.0001$ forall; $\mathrm{Q} 1$ vs. Q2: $\mathrm{P}<0.0001$; Q3 vs. Q2: $\mathrm{P}<0.0001$; $\mathrm{Q} 4$ vs. $\mathrm{Q} 2: \mathrm{P}=0.003)$.

Actually, the associationbetween leukocyte count and risk of adverse outcomes followed a U-shaped curve, with increased risk above and below the reference range of 5.5 to $6.7 \times 10^{9} / 1(\mathrm{Q} 2)$ (Fig. 3). The results of the Cox proportional hazards models illustrating the relationshipbetween leukocyte countand long-term clinical outcomes are shown in Table 2 and Additional file 1: Table S1-S4. As shown in Table 2, leukocyte count was an independent risk factor for all-cause death after multivariable adjustment $(\mathrm{P}<0.001)$. And the participants with the lowest leukocyte count $(\mathrm{Q} 1)$ and the highest leukocyte count(Q4) had higher risk of all-cause death compared with participants with leukocyte count range from $5.5 \times 10^{9} / 1$ to $6.7 \times 10^{9} / \mathrm{l}$.(Q1 vs. Q2: adjusted HR1.439, 95\%CI:1.060 to $1.953, \mathrm{P}=0.020$; $\mathrm{Q} 4$ vs. Q2: adjusted HR1.901, 95\%CI:1.424 to $2.539, \mathrm{P}<0.001$ ).

Interestingly, subgroup analyses of female participants confirmed the U-shaped relationship between leukocyte count and all-cause death (Table $3, \mathrm{P}=0.002$ ). However, despite a similar trend in male participants, there is no significant difference between groups. The subgroup analysis indicated the prognostic value of leukocyte count for all-cause death maybe different in different sexs. And female may contribute more to the relationship between leukocyte count and all-cause death.

After multivariable adjustment (Additional file 1: Table 1), therisk of compositecardiovascular events increased in patients withleukocyte count at Q3(HR, 1606; 95\%CI, 1.407to 1.904), Q4(HR, $1.650 ; 95 \% \mathrm{CI}$, 1.108to2.459) compared with patients with leukocyte count at Q2. Although similar trend was found in patients with leukocyte count at Q1, there was no statistical difference. Subgroup analysis by sex only found similar trend without statistical significance (Additional file 1: Table 2).Besides, after multivariable adjustment, participants with higher or lower leukocyte count at Q4 or Q1 did not have an increased risk for hospitalization for heart failure compared with patients with leukocyte count at Q2, and subgroup analysis reach a consistent result (Additional file 1: table s3 and table s4). Above results indicated that leukocyte count was not a prognostic factor for compositecardiovascular events and hospitalization for heart failure.

\section{Discussion}

This study found that the associationbetween leukocyte count and the risk ofadverse outcomes followed a U-shaped curve. Both lower and higher leukocyte count is related to a higher risk of adverse outcomes in the TOPCAT patientscohort.

Several studies have reported that pro-inflammatory biomarkers including high sensitivity C-reactive protein, tumor necrosis factor- $\alpha$, interleukin $6 / 8$, monocyte chemoattractant protein- 1 and pentraxin 3 were significantly increased in patients with HFpEF [14, 20-22].Consistent with previous studies, our results once again confirm that inflammatory responses may play an important role in the progression and development of HFpEF [20, 21, 23]. 
Table 1 Baseline characteristics $(n=3421)$

\begin{tabular}{|c|c|c|c|c|c|}
\hline \multirow[t]{2}{*}{ Characteristic } & \multicolumn{5}{|c|}{ Leukocyte count } \\
\hline & $\leqq 5.5 n=753$ & $5.5-6.7 n=707$ & $6.7-8.0 n=720$ & $>8.0 n=718$ & p-value \\
\hline Age, mean $\pm S D$, years & $69 \pm 9.2$ & $69 \pm 9.7$ & $69 \pm 10$ & $69 \pm 9$ & 0.867 \\
\hline Male (\%) & $289(38)$ & $304(43)$ & $362(50)$ & $372(52)$ & 0.000 \\
\hline Race & & & & & 0.620 \\
\hline White (\%) & $671(89)$ & $629(89)$ & $641(89)$ & $629(88)$ & \\
\hline Black (\%) & $69(9)$ & $58(8)$ & $63(9)$ & $66(9)$ & \\
\hline Other (\%) & $13(2)$ & $20(2)$ & $16(2)$ & $23(3)$ & \\
\hline Smoker (\%) & $237(32)$ & $241(34)$ & $267(37)$ & $306(43)$ & 0.001 \\
\hline Hypertension (\%) & $685(91)$ & $645(91)$ & $673(94)$ & $673(94)$ & 0.077 \\
\hline Dyslipidemia (\%) & $431(57)$ & $406(57)$ & $423(59)$ & $483(67)$ & 0.000 \\
\hline Previous myocardial infarction (\%) & $143(19)$ & $154(22)$ & $173(24)$ & $192(27)$ & 0.004 \\
\hline Prior heart failure hospitalization (\%) & $562(75)$ & $511(72)$ & $520(72)$ & $504(70)$ & 0.304 \\
\hline Angina pectoris (\%) & $340(45)$ & $347(49)$ & $345(48)$ & $311(43)$ & 0.112 \\
\hline $\mathrm{PCl}(\%)$ & $89(12)$ & $87(12)$ & $97(14)$ & $132(19)$ & 0.000 \\
\hline CABG (\%) & $75(10)$ & $80(11)$ & $85(12)$ & $113(16)$ & 0.006 \\
\hline Diabetes mellitus (\%) & $198(26)$ & $198(28)$ & $244(34)$ & $318(44)$ & 0.000 \\
\hline Atrial fibrillation (\%) & $262(35)$ & $218(31)$ & $239(33)$ & $280(39)$ & 0.011 \\
\hline COPD $(\%)$ & $58(8)$ & $67(10)$ & $89(12)$ & $124(17)$ & 0.000 \\
\hline Asthma (\%) & $36(5)$ & $56(8)$ & $43(6)$ & $61(9)$ & 0.016 \\
\hline Stroke $(\%)$ & $56(7)$ & $43(6)$ & $59(8)$ & $68(10)$ & 0.112 \\
\hline Peripheral arterial disease (\%) & $49(7)$ & $55(8)$ & $66(9)$ & $89(12)$ & 0.000 \\
\hline Thyroid disease (\%) & $128(17)$ & $105(15)$ & $104(15)$ & $143(20)$ & 0.021 \\
\hline Pacemaker implanted (\%) & $64(9)$ & $50(7)$ & $56(8)$ & $61(9)$ & 0.713 \\
\hline $\operatorname{ICD}(\%)$ & $10(1.3)$ & $8(1.1)$ & $8(1.1)$ & $12(1.7)$ & 0.773 \\
\hline HR (b.p.m.) & $69 \pm 10.1$ & $68 \pm 9.9$ & $68 \pm 11.1$ & $70 \pm 11.3$ & 0.078 \\
\hline Systolic blood pressure, mean $\pm S D, m m H g$ & $129 \pm 12.6$ & $130 \pm 13.9$ & $130 \pm 14.6$ & $129 \pm 14.9$ & 0.110 \\
\hline Diastolic blood pressure & $76 \pm 10.4$ & $77 \pm 10.6$ & $76 \pm 10.8$ & $74 \pm 11.1$ & 0.000 \\
\hline Body mass index, mean $\pm \mathrm{SD}, \mathrm{kg} / \mathrm{m}^{2}$ & $31 \pm 6.6$ & $32 \pm 6.5$ & $32 \pm 7.1$ & $34 \pm 7.9$ & 0.000 \\
\hline $\mathrm{eGFR}(\mathrm{mL} / \mathrm{min})$ & $67 \pm 18.2$ & $69 \pm 22.5$ & $68 \pm 19.8$ & $65 \pm 20.1$ & 0.002 \\
\hline $\mathrm{BUN}(\mathrm{mg} / \mathrm{dL})$ & $16.5(6.8,22.1)$ & $16.2(5.0,22.4)$ & $16.5(5.6,23.0)$ & $17.6(8.1,26.0)$ & 0.004 \\
\hline Hematocrit (\%) & $39 \pm 5.0$ & $40 \pm 4.8$ & $40 \pm 5.4$ & $41 \pm 5.7$ & 0.000 \\
\hline Hemoglobin (g/dL) & $12.9(12.0,14.0)$ & $13.2(12.2,14.3)$ & $13.4(12.3,14.5)$ & $13.5(12.2,14.8)$ & 0.000 \\
\hline Platelet (k/uL) & $207(173,243)$ & $220(188,254)$ & $223(193,264)$ & $245(208,294)$ & 0.000 \\
\hline Albumin (g/dL) & $3.9 \pm 2.5$ & $3.8 \pm 2.7$ & $3.7 \pm 2.5$ & $3.7 \pm 2.8$ & 0.000 \\
\hline $\log B N P$ & $2.6 \pm 0.5$ & $2.6 \pm 0.5$ & $2.6 \pm 0.5$ & $2.6 \pm 0.5$ & 0.627 \\
\hline $\operatorname{LVEF}(\%)$ & $59 \pm 6.5$ & $59 \pm 6.9$ & $59 \pm 6.0$ & $59 \pm 6.7$ & 0.076 \\
\hline New York Heart Association class III-IV (\%) & $514(68)$ & $509(72)$ & $501(70)$ & $428(60)$ & 0.000 \\
\hline Aspirin use (\%) & $453(60)$ & $458(65)$ & $475(66)$ & $458(64)$ & 0.110 \\
\hline b-blockers (\%) & $573(76)$ & $555(79)$ & $565(79)$ & $551(78)$ & 0.599 \\
\hline ACEi (\%) & $504(66)$ & $455(64)$ & $455(63)$ & $438(61)$ & 0.120 \\
\hline ARB (\%) & $107(14)$ & $113(16)$ & $109(15)$ & $132(18)$ & 0.155 \\
\hline Statins (\%) & $334(44)$ & $332(47)$ & $362(50)$ & $426(59)$ & 0.000 \\
\hline Calcium channel blockers (\%) & $276(37)$ & $292(41)$ & $272(38)$ & $281(39)$ & 0.300 \\
\hline Spironolactone (\%) & $361(48)$ & $370(52)$ & $346(48)$ & $378(53)$ & 0.118 \\
\hline Loop diuretic (\%) & $326(43)$ & $329(47)$ & $349(49)$ & $458(64)$ & 0.000 \\
\hline Thiazide diuretic (\%) & $322(43)$ & $278(39)$ & $286(40)$ & $216(30)$ & 0.000 \\
\hline
\end{tabular}

Values are presented as mean \pm SD or median (25th-75th percentile) for continuous variables and number (\%) for categorical variables. Statistical significance for continuous data was tested using the analysis of variance procedure and categorical data was tested using the $x^{2}$ test

ACEl, angiotensin-converting enzyme inhibitor; ARB, angiotensin receptor blocker; BMI, body mass index; BNP, B-type natriuretic peptide; BUN, blood urea nitrogen; ICD, Implantable Cardioverter Defibrillator; COPD, chronic obstructive pulmonary disease; $\mathrm{CABG}$, Coronary Artery Bypass Grafting;PCl, percutaneous coronary intervention;DBP,diastolic blood pressure; eGFR, estimated glomerular filtration rate; HR, heart rate; LVEF, left ventricular ejection fraction; NYHA, New York Heart Association; SBP, systolic blood pressure

eGFR by the Chronic Kidney Disease Epidemiology Collaboration formula 
Table 1 (continued)

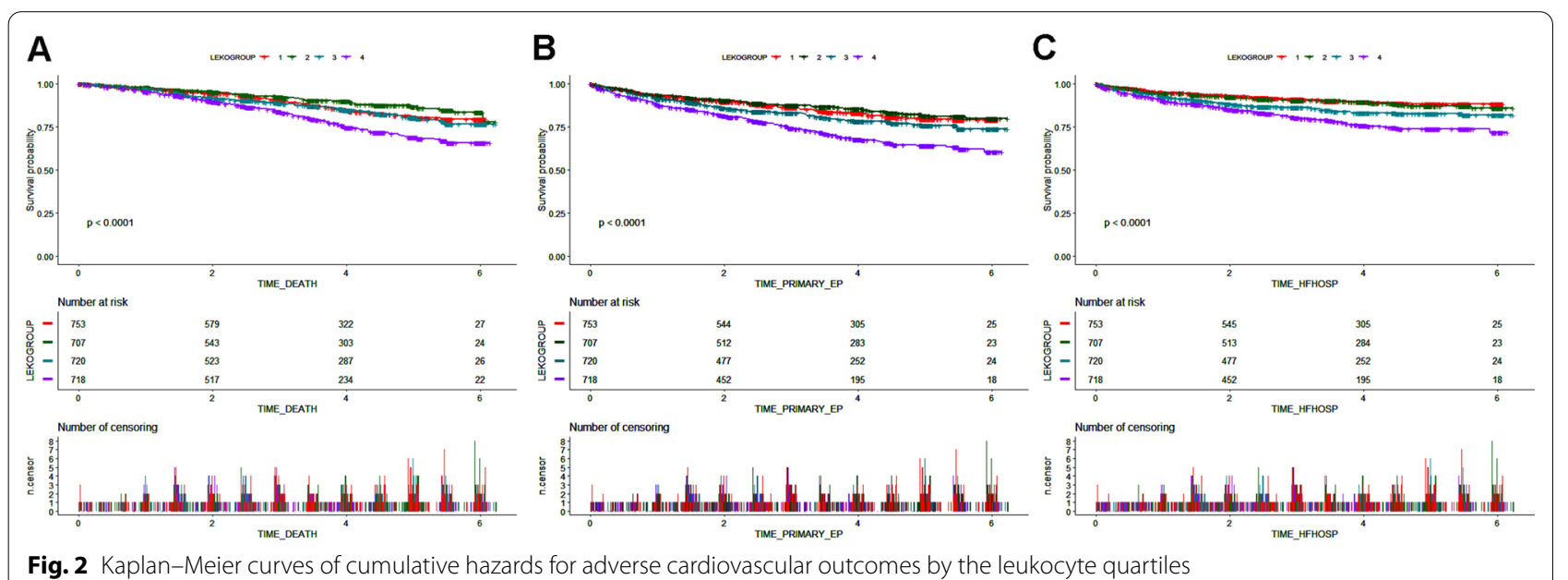

Fig. 2 Kaplan-Meier curves of cumulative hazards for adverse cardiovascular outcomes by the leukocyte quartiles

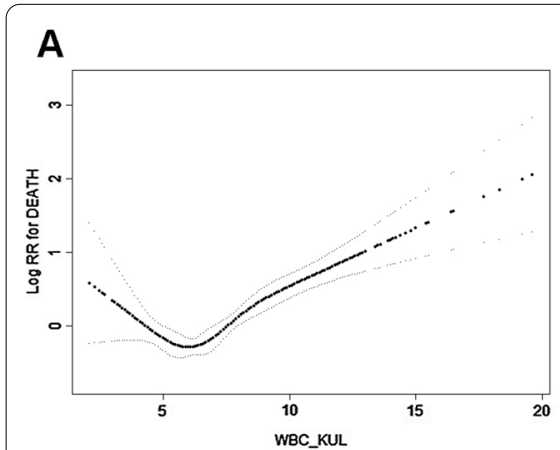

Fig. 3 Restricted Cubic Spline of the Association of leukocyte With Risk of adverse cardiovascular outcomes in HFpEF. This figure is as the Central Illustration of our study, which shows a U-shaped relationship between leukocyte count and adverse outcomes in patients with HFpEF.
However, although leukocyte count acts as an important marker for inflammation level in body, few previous studies have assessed the association between leukocyte countand cardiovascular events in patients with HFpEF. Previousstudies only showed that the prognosticvalue of relative lymphocyte count in patients with chronic HFrEF [12, 24-26].In further, high leukocyte countwas found to be associated with increased long-term incidence of HFhospitalizationsin middle-aged men [10].Besides, Kim et al. found that neutrophil-to-lymphocyte ratiowas prospectively associatedwith heart failure [5]. In line with above studies, present finding indicates that leukocyte countisassociated with both all-causedeath and composite cardiovascular events specifically in HFpEF patients, reaffirming this important link between leukocytecount and heart failure regardless of ejection fraction. Recently, Bajaj NS et al. [27]did a similar study and they found that leucocyte count $>7100$ cells $/ \mu \mathrm{L}$ was independently associated with adverse clinical outcomes especially
HF hospitalization in HFpEF patients from TOPCATAmericas.In our study, we focused on the whole population in TOPCAT study and patients with LVEF $<50 \%$ were excluded, which may be attributed to the different result from the study by Bajaj NS. In our study, we found a U-shaped relationship between the risk of clinical outcomes especially all-cause death and leukocyte count. Besides, the subgroup analysis showed that female may contribute more to such relationship of leukocyte count and all-cause death. However, the U-shaped relationship also showed an increased risk of clinical outcomes for patients with higher leukocyte count in our study, which was confirmed by the study by Bajaj NS.Besides, although similar trend was found, leukocyte count was not a prognostic factor for compositecardiovascular events and hospitalization for heart failure in this study. This may be caused by the heterogeneity of HFpEF, the shortage of the second analysis and the limit sample volume. Further 
Table 2 Univariate and multivariable Cox regression analysis of all-cause mortality $(n=2898)$

\begin{tabular}{|c|c|c|c|c|c|c|}
\hline \multirow[t]{2}{*}{ All-cause mortality } & \multicolumn{3}{|c|}{ Univariate analysis } & \multicolumn{3}{|c|}{ Multivariate analysis } \\
\hline & HR & $95 \% \mathrm{Cl}$ & p-value & HR & $95 \% \mathrm{Cl}$ & p-value \\
\hline Age & 1.054 & $1.043-1.065$ & 0.000 & 1.046 & $1.033-1.059$ & 0.000 \\
\hline Sex & 0.67 & $0.554-0.810$ & 0.000 & 1.698 & $1.368-2.106$ & 0.000 \\
\hline \multirow[t]{3}{*}{ Race } & 1.528 & $1.251-1.867$ & 0.000 & & & 0.037 \\
\hline & & & & 0.531 & $0.326-0.865$ & 0.011 \\
\hline & & & & 0.591 & $0.332-1.049$ & 0.073 \\
\hline BMl & 1.007 & $0.993-1.021$ & 0.330 & - & - & - \\
\hline Smoker & 1.170 & $1.037-1.320$ & 0.011 & - & - & - \\
\hline LVEF & 0.998 & $0.984-1.013$ & 0.820 & - & - & - \\
\hline Angina pectoris & 0.613 & $0.504-0.745$ & 0.000 & 0.815 & $0.653-1.017$ & 0.071 \\
\hline Prior heart failure hospitalization & 0.810 & $0.657-0.997$ & 0.047 & 1.124 & $0.901-1.403$ & 0.301 \\
\hline Previous myocardial infarction & 1.266 & $1.026-1.563$ & 0.028 & 0.777 & $0.603-1.002$ & 0.052 \\
\hline Stroke & 1.558 & $1.151-2.110$ & 0.004 & 0.940 & $0.686-1.289$ & 0.702 \\
\hline CABG & 1.655 & $1.293-2.118$ & 0.000 & 1.066 & $0.800-1.422$ & 0.661 \\
\hline $\mathrm{PCl}$ & 1.483 & $1.161-1.893$ & 0.002 & 1.061 & $0.803-1.403$ & 0.677 \\
\hline COPD & 1.629 & $1.257-2.111$ & 0.000 & 0.936 & $0.713-1.228$ & 0.634 \\
\hline Asthma & 1.601 & $1.152-2.226$ & 0.005 & 0.812 & $0.574-1.148$ & 0.239 \\
\hline Hypertension & 0.815 & $0.586-1.133$ & 0.223 & - & - & - \\
\hline Peripheral arterial disease & 2.154 & $1.669-2.779$ & 0.000 & 0.615 & $0.468-0.809$ & 0.001 \\
\hline Dyslipidemia & 1.271 & $1.043-1.550$ & 0.018 & 1.105 & $0.857-1.426$ & 0.441 \\
\hline ICD & 1.605 & $0.797-3.230$ & 0.185 & - & - & - \\
\hline Pacemaker & 1.983 & $1.500-2.621$ & 0.000 & 0.978 & $0.724-1.320$ & 0.884 \\
\hline Atrial fibrillation & 1.530 & $1.264-1.851$ & 0.000 & 1.016 & $0.821-1.258$ & 0.884 \\
\hline Thyroid disease & 1.219 & $0.957-1.553$ & 0.108 & - & - & - \\
\hline Diabetes mellitus & 0.595 & $0.491-0.721$ & 0.000 & 0.857 & $0.685-1.071$ & 0.175 \\
\hline Heart rate & 1.017 & $1.008-1.026$ & 0.000 & 1.021 & $1.012-1.031$ & 0.000 \\
\hline Systolic blood pressure & 0.981 & $0.974-0.988$ & 0.000 & 0.992 & $0.984-1.000$ & 0.050 \\
\hline Diastolic blood pressure & 0.959 & $0.951-0.967$ & 0.000 & 0.994 & $0.982-1.006$ & 0.300 \\
\hline Fasting glucose & 1.002 & $0.998-1.005$ & 0.343 & - & - & - \\
\hline New York Heart Association class III-IV & 1.723 & $1.423-2.086$ & 0.000 & 0.806 & $0.658-0.988$ & 0.038 \\
\hline eGFR & 0.979 & $0.973-0.984$ & 0.000 & 0.994 & $0.988-1.000$ & 0.055 \\
\hline Leukocyte group & 1.249 & $1.146-1.361$ & 0.000 & & & 0.000 \\
\hline 1 & & & & 1.439 & $1.060-1.953$ & 0.020 \\
\hline 2 & & & & Reference & & \\
\hline 3 & & & & 1.510 & $1.113-2.050$ & 0.008 \\
\hline 4 & & & & 1.901 & $1.424-2.539$ & 0.000 \\
\hline Hemoglobin & 0.833 & $0.786-0.882$ & 0.000 & 0.898 & $0.843-0.958$ & 0.001 \\
\hline BUN & 1.030 & $1.025-1.036$ & 0.000 & 1.009 & $1.001-1.017$ & 0.023 \\
\hline Albumin & 0.983 & $0.945-1.023$ & 0.411 & - & - & - \\
\hline Aspirin & 1.301 & $1.074-1.576$ & 0.007 & 1.089 & $0.884-1.341$ & 0.424 \\
\hline b-blockers & 1.16 & $0.915-1.471$ & 0.220 & - & - & - \\
\hline ACEi & 1.355 & $1.116-1.643$ & 0.002 & 0.945 & $0.770-1.160$ & 0.591 \\
\hline ARB & 0.862 & $0.670-1.109$ & 0.248 & - & - & - \\
\hline Statin & 0.726 & $0.599-0.878$ & 0.001 & 1.072 & $0.837-1.372$ & 0.581 \\
\hline Loop diuretic & 0.304 & $0.245-0.377$ & 0.000 & 0.553 & $0.423-0.724$ & 0.000 \\
\hline Thiazide Diuretic & 0.494 & $0.398-0.612$ & 0.000 & 1.080 & $0.840-1.388$ & 0.548 \\
\hline Spironolactone & 1.029 & $0.851-1.243$ & 0.769 & - & - & - \\
\hline
\end{tabular}

$\mathrm{Cl}$ : confidence interval; HR: hazard ratio 
Table 3 Subgroup analysis of Cox proportional-hazards model divided by sex for All-cause mortality

\begin{tabular}{|c|c|c|c|c|c|c|}
\hline \multirow[t]{2}{*}{ All-cause mortality } & \multicolumn{3}{|l|}{ Male } & \multicolumn{3}{|c|}{ Female } \\
\hline & HR & $95 \% \mathrm{Cl}$ & $p$-value & HR & $95 \% \mathrm{Cl}$ & p-value \\
\hline Age & 1.047 & $1.029-1.066$ & 0.000 & 1.038 & $1.019-1.057$ & 0.000 \\
\hline \multirow[t]{3}{*}{ Race } & & & 0.473 & & & 0.007 \\
\hline & 0.684 & $0.328-1.424$ & 0.310 & 0.344 & $0.175-0.676$ & 0.002 \\
\hline & 0.837 & $0.354-1.982$ & 0.687 & 0.319 & $0.143-0.709$ & 0.005 \\
\hline Smoker & 0.858 & $0.743-0.991$ & 0.037 & 0.864 & $0.697-1.070$ & 0.180 \\
\hline Angina pectoris & 0.967 & $0.715-1.309$ & 0.830 & 0.692 & $0.493-0.970$ & 0.033 \\
\hline Prior heart failure hospitalization & 1.269 & $0.933-1.727$ & 0.130 & 0.928 & $0.665-1.296$ & 0.661 \\
\hline Previous myocardial infarction & 0.742 & $0.536-1.025$ & 0.071 & 0.893 & $0.583-1.366$ & 0.602 \\
\hline Stroke & 0.851 & $0.551-1.315$ & 0.468 & 0.993 & $0.619-1.593$ & 0.978 \\
\hline CABG & 1.109 & $0.772-1.592$ & 0.576 & 0.953 & $0.580-1.566$ & 0.850 \\
\hline $\mathrm{PCl}$ & 1.168 & $0.805-1.693$ & 0.414 & 0.927 & $0.594-1.447$ & 0.739 \\
\hline COPD & 1.118 & $0.780-1.602$ & 0.544 & 0.687 & $0.445-1.061$ & 0.091 \\
\hline Asthma & 0.602 & $0.356-1.018$ & 0.058 & 1.070 & $0.660-1.736$ & 0.783 \\
\hline Peripheral arterial disease & 0.562 & $0.394-0.801$ & 0.001 & 0.657 & $0.420-1.029$ & 0.067 \\
\hline Dyslipidemia & 1.158 & $0.816-1.643$ & 0.412 & 1.121 & $0.770-1.634$ & 0.550 \\
\hline Pacemaker & 0.863 & $0.574-1.298$ & 0.479 & 1.116 & $0.697-1.787$ & 0.647 \\
\hline Atrial fibrillation & 1.139 & $0.852-1.521$ & 0.380 & 0.860 & $0.622-1.189$ & 0.360 \\
\hline Diabetes mellitus & 0.986 & $0.729-1.334$ & 0.926 & 0.737 & $0.527-1.032$ & 0.075 \\
\hline Heart rate & 1.019 & $1.006-1.032$ & 0.005 & 1.028 & $1.014-1.042$ & 0.000 \\
\hline Systolic blood pressure & 0.993 & $0.982-1.005$ & 0.244 & 0.994 & $0.982-1.005$ & 0.286 \\
\hline Diastolic blood pressure & 0.990 & $0.974-1.007$ & 0.251 & 0.992 & $0.975-1.010$ & 0.332 \\
\hline New York Heart Association class III-IV & 0.805 & $0.604-1.072$ & 0.138 & 0.756 & $0.557-1.026$ & 0.072 \\
\hline eGFR & 0.995 & $0.986-1.003$ & 0.211 & 0.993 & $0.984-1.002$ & 0.143 \\
\hline Leukocyte group & & & 0.088 & & & 0.002 \\
\hline 1 & 1.134 & $0.745-1.726$ & 0.557 & 1.907 & $1.188-3.059$ & 0.007 \\
\hline 2 & reference & & & & & \\
\hline 3 & 1.150 & $0.768-1.721$ & 0.498 & 2.088 & $1.291-3.375$ & 0.003 \\
\hline 4 & 1.571 & $1.071-2.303$ & 0.021 & 2.445 & $1.543-3.875$ & 0.000 \\
\hline Hemoglobin & 0.889 & $0.816-0.968$ & 0.007 & 0.910 & $0.822-1.006$ & 0.066 \\
\hline BUN & 1.011 & $1.001-1.021$ & 0.032 & 1.005 & $0.993-1.018$ & 0.419 \\
\hline Aspirin & 1.354 & $1.021-1.795$ & 0.035 & 0.838 & $0.609-1.153$ & 0.277 \\
\hline ACEi & 1.007 & $0.759-1.335$ & 0.963 & 0.884 & $0.650-1.202$ & 0.432 \\
\hline Statin & 1.006 & $0.713-1.420$ & 0.972 & 1.142 & $0.790-1.651$ & 0.479 \\
\hline Loop Diuretic & 0.627 & $0.441-0.892$ & 0.010 & 0.467 & $0.308-0.707$ & 0.000 \\
\hline Thiazide Diuretic & 0.925 & $0.666-1.285$ & 0.642 & 1.303 & $0.880-1.930$ & 0.186 \\
\hline
\end{tabular}

well-designed study was warranted to investigate the actual role of leukocyte in patients with HFpEF.

Although the association between leukocyte and heart failure is strongly supported by current clinical evidences [26]. It is not known whether leukocytes are involved directly in the pathogenesis of heart failure or areonly accompany with the disease.Severalsystemic proinflammatory conditions including obesity, hypertension, diabetes or metabolic syndrome were usually combined in patients with HFpEF,whichmight be the fundamental mechanism that leads to inflammation andoxidative stress [28]. The increased pro-inflammatory state and oxidativestress may in turn result incoronary microvascular endothelial dysfunction and myocardialfibrosis, consequently leading to adverse cardiovascular events finally. This may explain the increased risk of adverse outcomes ofHFpEF patients with higher level of leukocyte count in this study.

However, in our study, we presented a U-shaped relationship between leukocyte count and the risk of adverse outcomes, indicatingmore complex mechanisms might be involved underling the relationship between leukocyte 
level and cardiovascular outcomes in HFpEF patients. Leukocytescan not only facilitate the proteolysis of the collagen matrix but also promote interstitial myocardial fibrosis, which eventually contribute tothe cardiac remodeling and heart failure [4]. Confirming this,recent study demonstratedthat by activating fibroblasts and stimulating collagen deposition, IL-10 derived from $\mathrm{T}$ cellsand macrophagescan induce myocardial stiffness and impair myocardial relaxation $[29,30]$. But on the other hand, through secretion of angiogenesis-promoting cytokines, leukocytescan also protect the nonischemic remote myocardium in ischemic heart disease [4]. This indicates thattoo lessleukocyte may be harmful for some heart disease.

In addition, the U-shaped relationship between leukocyte count and the risk of adverse cardiovascular outcomes persisted even aftercontrolling for baseline covariates.The U-shaped relationship may also be a potential reason for the unsuccessful clinical trials attempting to combat HFby blocking inflammation [11]. Although canakinumabis related to a dose-dependent reduction in heart failure relatedhospitalization and the composite of heart failure-related mortality and hospitalization, it is not efficient in all population but patients with elevatedhsCRP [31].Besides,interaction between inflammation and body weight, blood pressure, and blood glucose might jointly affect theoutcomes of HFpEF patients and the sum of the complex interaction may bealso responsible for the observedU-shaped relationshipin this study [32-35].

\section{Conclusions}

In this study, we found a U-shaped relationship between leukocyte count and risk of clinical outcomes, and subgroup analysis showed that female contributed more to such relationship for all-cause death. Both higher and lower leukocyte count are associated with worse outcomes in patients with HFpEF, which may be attributed to the two sides of inflammation in cardiac remodeling.

\section{Limitations}

The findings of this study must be interpreted in the contextof limitations inherent to the TOPCAT studydesign. First, there is heterogeneityin HFpEF,so these findings may not represent all theHFpEF classifications. Secondly, we cannot exclude biasintroduced by leukocyte levels measured at laboratories and there is lack of CRP value and serial measurements about leukocyte count in the database, which limit the strength of the conclusion. Thirdly, leukocyte count is elevated or decreased commonly in patient with acute infection or blood system diseases, no information is applied about the exclusion of such patients in the TOPCAT trial, the impact of acute infection or blood system diseases thus remain unknown and served as a limitation of present analysis.At last, although the subtype of leukocyte may play pivotal role in cardiovascular disease, we did not assess the specific role due to the unavailability of the related information in the present database.

\begin{abstract}
Abbreviations
HFpEF: Heart failure with preserved ejection fraction; BMI: Body mass index blood; BUN: Urea nitrogen; BNP: Brain natriuretic peptide; CKD-EPI: Chronickidney disease epidemiology collaboration; ACEI/ARB: Angiotensin-converting enzyme inhibitor/Angiotensin receptor blocker; ICD: Implantable cardioverter defibrillators; PCI: Percutaneous coronary intervention; CABG: Coronary artery bypass graft; COPD: Chronic obstructive pulmonary disease; LVEF: Left ventricular ejection fraction.
\end{abstract}

\section{Supplementary Information}

The online version contains supplementary material available at https://doi. org/10.1186/s12872-021-02142-y.

Additional file 1. Supplemental Table 1. Univariate and multivariable Cox regression analysis of Composite cardiovascular events $(n=2898)$. Supplemental Table 2. Subgroup analysis of Cox proportional-hazards model divided by gender for Composite cardiovascular events ( $n=2898$ ). Supplemental Table 3. Univariate and multivariable Cox regression analysis of hospitalization for heart failure $(n=2898)$. Supplemental Table 4. Subgroup analysis of Cox proportional-hazards model divided by gender for Hospitalization for heart failure $(n=2898)$

\section{Acknowledgements}

This manuscript was prepared using TOPCAT Research Materialsobtained from the National Heart, Lung, and BloodInstitute.

\section{Authors' contributions}

$\mathrm{ZZ}$ and SZ analyzed the data and wrote the main manuscript text. All authors read and approved the final manuscript.

\section{Funding}

This work was supported in part by the National Science Foundation of China (NSFC) Projects 81600248 (to Z Zhu), Hunan Provincial Natural Science Foundation of China Projects 2018JJ3744 (to Z Zhu).The above funding bodies played no role in the design of the study and collection, analysis, and interpretation of data and in writing the manuscript.

Availability of data and materials

The datasets used and/or analysed during the current study are available from the corresponding author on reasonable request.

\section{Declarations}

\section{Ethics approval and consent to participate}

The study was approved by the ethics committee of The Second Xiangya Hospital and obeyed the Declaration of Helsinki (No. 2017YFC0908802). All patients have provided written consent to participate in this study.

\section{Consent to publication}

Not applicable.

\section{Competing interests}

The authors declare that they have no competing interests. 
Received: 28 October 2020 Accepted: 29 June 2021

Published online: 07 July 2021

\section{References}

1. Borlaug BA, Paulus WJ. Heart failure with preserved ejection fraction: pathophysiology, diagnosis, and treatment. Eur Heart J. 2011;32(6):670-9.

2. Borlaug BA, Anstrom KJ, Lewis GD, Shah SJ, Levine JA, Koepp GA, Givertz MM, Felker GM, LeWinter MM, Mann DL, et al. Effect of inorganic nitrite vs placebo on exercise capacity among patients with heart failure with preserved ejection fraction: the INDIE-HFpEF randomized clinical trial. JAMA. 2018;320(17):1764-73.

3. Upadhya B, Haykowsky MJ, Kitzman DW: Therapy for heart failure with preserved ejection fraction: current status, unique challenges, and future directions. Heart failure reviews 2018.

4. Swirski FK, Nahrendorf M. Leukocyte behavior in atherosclerosis, myocardial infarction, and heart failure. Science. 2013:339(6116):161-6.

5. Kim S, Eliot M, Koestler DC, Wu WC, Kelsey KT. Association of neutrophilto-lymphocyte ratio with mortality and cardiovascular disease in the Jackson heart study and modification by the Duffy antigen variant. JAMA Cardiol. 2018;3(6):455-62.

6. Madjid M, Awan I, Willerson JT, Casscells SW. Leukocyte count and coronary heart disease: implications for risk assessment. J Am Coll Cardiol. 2004;44(10):1945-56.

7. Mann DL. Inflammatory mediators and the failing heart: past, present, and the foreseeable future. Circ Res. 2002;91(11):988-98.

8. Mann DL. Innate immunity and the failing heart: the cytokine hypothesis revisited. Circ Res. 2015;116(7):1254-68.

9. Nymo SH, Hulthe J, Ueland T, McMurray J, Wikstrand J, Askevold ET, Yndestad A, Gullestad L, Aukrust P. Inflammatory cytokines in chronic heart failure: interleukin- 8 is associated with adverse outcome. Results from CORONA. Eur J Heart Fail. 2014;16(1):68-75.

10. Engstrom G, Melander O, Hedblad B. Leukocyte count and incidence of hospitalizations due to heart failure. Circ Heart Fail. 2009:2(3):217-22.

11. Hofmann U, Frantz S. How can we cure a heart "in flame"? A translational view on inflammation in heart failure. Basic Res Cardiol. 2013;108(4):356.

12. Huehnergarth KV, Mozaffarian D, Sullivan MD, Crane BA, Wilkinson CW, Lawler RL, McDonald GB, Fishbein DP, Levy WC. Usefulness of relative lymphocyte count as an independent predictor of death/urgent transplant in heart failure. Am J Cardiol. 2005;95(12):1492-5

13. Everett BM, Cornel JH, Lainscak M, Anker SD, Abbate A, Thuren T, Libby P, Glynn RJ, Ridker PM. Anti-Inflammatory therapy with canakinumab for the prevention of hospitalization for heart failure. Circulation. 2019;139(10):1289-99.

14. de Boer RA, Nayor M, deFilippi CR, Enserro D, Bhambhani V, Kizer JR, Blaha MJ, Brouwers FP, Cushman M, Lima JAC, et al. Association of cardiovascular biomarkers with incident heart failure with preserved and reduced ejection fraction. JAMA Cardiol. 2018;3(3):215-24.

15. Paulus WJ, Tschope C. A novel paradigm for heart failure with preserved ejection fraction: comorbidities drive myocardial dysfunction and remodeling through coronary microvascular endothelial inflammation. J Am Coll Cardiol. 2013:62(4):263-71.

16. Pitt B, Pfeffer MA, Assmann SF, Boineau R, Anand IS, Claggett B, Clausell N, Desai AS, Diaz R, Fleg JL, et al. Spironolactone for heart failure with preserved ejection fraction. N Engl J Med. 2014;370(15):1383-92.

17. Desai AS, Lewis EF, Li R, Solomon SD, Assmann SF, Boineau R, Clausell N, Diaz R, Fleg JL, Gordeev I, et al. Rationale and design of the treatment of preserved cardiac function heart failure with an aldosterone antagonist trial: a randomized, controlled study of spironolactone in patients with symptomatic heart failure and preserved ejection fraction. Am Heart J. 2011;162(6):966-72

18. Ponikowski P, Voors AA, Anker SD, Bueno H, Cleland JGF, Coats AJS, Falk V, Gonzalez-Juanatey JR, Harjola VP, Jankowska EA, et al. 2016 ESC Guidelines for the diagnosis and treatment of acute and chronic heart failure: the Task Force for the diagnosis and treatment of acute and chronic heart failure of the European Society of Cardiology (ESC)Developed with the special contribution of the Heart Failure Association (HFA) of the ESC. Eur Heart J. 2016;37(27):2129-200.

19. Levey AS, Stevens LA, Schmid CH, Zhang YL, Castro AF 3rd, Feldman HI, Kusek JW, Eggers P, Van Lente F, Greene T, et al. A new equation to estimate glomerular filtration rate. Ann Intern Med. 2009;150(9):604-12.

20. Cheng JM, Akkerhuis KM, Battes LC, van Vark LC, Hillege HL, Paulus WJ, Boersma E, Kardys I. Biomarkers of heart failure with normal ejection fraction: a systematic review. Eur J Heart Fail. 2013;15(12):1350-62.

21. D'Elia E, Vaduganathan M, Gori M, Gavazzi A, Butler J, Senni M. Role of biomarkers in cardiac structure phenotyping in heart failure with preserved ejection fraction: critical appraisal and practical use. Eur J Heart Fail. 2015;17(12):1231-9.

22. Collier P, Watson CJ, Voon V, Phelan D, Jan A, Mak G, Martos R, Baugh JA, Ledwidge MT, McDonald KM. Can emerging biomarkers of myocardial remodelling identify asymptomatic hypertensive patients at risk for diastolic dysfunction and diastolic heart failure? Eur J Heart Fail. 2011;13(10):1087-95.

23. Glezeva N, Baugh JA. Role of inflammation in the pathogenesis of heart failure with preserved ejection fraction and its potential as a therapeutic target. Heart Fail Rev. 2014;19(5):681-94.

24. Ommen SR, Hodge DO, Rodeheffer RJ, McGregor CG, Thomson SP, Gibbons RJ. Predictive power of the relative lymphocyte concentration in patients with advanced heart failure. Circulation. 1998;97(1):19-22.

25. Acanfora D, Gheorghiade M, Trojano L, Furgi G, Pasini E, Picone C, Papa A, lannuzzi GL, Bonow RO, Rengo F. Relative lymphocyte count: a prognostic indicator of mortality in elderly patients with congestive heart failure. Am Heart J. 2001:142(1):167-73.

26. Strassheim D, Dempsey EC, Gerasimovskaya E, Stenmark K, Karoor V. Role of inflammatory cell subtypes in heart failure. J Immunol Res. 2019;2019:2164017

27. Bajaj NS, Kalra R, Gupta K, Aryal S, Rajapreyar I, Lloyd SG, McConathy J, Shah SJ, Prabhu SD. Leucocyte count predicts cardiovascular risk in heart failure with preserved ejection fraction: insights from TOPCAT Americas. ESC Heart Fail. 2020;7(4):1676-87.

28. Redfield MM. Heart failure with preserved ejection fraction. N Engl J Med. 2016:375(19):1868-77.

29. Hulsmans M, Sager HB, Roh JD, Valero-Munoz M, Houstis NE, Iwamoto Y, Sun Y, Wilson RM, Wojtkiewicz G, Tricot B, et al. Cardiac macrophages promote diastolic dysfunction. J Exp Med. 2018;215(2):423-40.

30. Kallikourdis M, Martini E, Carullo P, Sardi C, Roselli G, Greco CM, Vignali D, Riva F, Ormbostad Berre AM, Stolen TO, et al. T cell costimulation blockade blunts pressure overload-induced heart failure. Nat Commun. 2017;8:14680

31. Everett BM, Cornel J, Lainscak M, Anker SD, Abbate A, Thuren T, Libby P, Glynn RJ, Ridker PM: Anti-Inflammatory Therapy with Canakinumab for the Prevention of Hospitalization for Heart Failure. Circulation 2018.

32. Currie CJ, Peters JR, Tynan A, Evans M, Heine RJ, Bracco OL, Zagar T, Poole $\mathrm{CD}$. Survival as a function of $\mathrm{HbA}(1 \mathrm{c})$ in people with type 2 diabetes: $\mathrm{a}$ retrospective cohort study. Lancet. 2010;375(9713):481-9.

33. Strandberg TE, Strandberg AY, Salomaa W, Pitkala KH, Tilvis RS, Sirola J, Miettinen TA. Explaining the obesity paradox: cardiovascular risk, weight change, and mortality during long-term follow-up in men. Eur Heart J. 2009:30(14):1720-7.

34. Bangalore S, Qin J, Sloan S, Murphy SA, Cannon CP. Investigators PI-TT: What is the optimal blood pressure in patients after acute coronary syndromes? Relationship of blood pressure and cardiovascular events in the PRavastatin OR atorVastatin Evaluation and Infection TherapyThrombolysis In Myocardial Infarction (PROVE IT-TIMI) 22 trial. Circulation. 2010;122(21):2142-51.

35. Vilaro JR, Ahmed M, Aranda JM. Heart failure with preserved ejection fraction: time to revisit the stiff heart. Cardiovascular Innovations and Applications. 2019;3(4):409-20.

\section{Publisher's Note}

Springer Nature remains neutral with regard to jurisdictional claims in published maps and institutional affiliations. 